\title{
Critical exponent for semilinear wave equation with critical potential
}

\author{
Xinfu Li
}

\begin{abstract}
We consider the Cauchy problem for the semilinear wave equation

$$
u_{t t}-\Delta u+V(x) u_{t}=|u|^{p} .
$$

When $V(x)=V_{0}\left(1+|x|^{2}\right)^{-1 / 2}, V_{0} \geq n$, we prove that the critical exponent for the problem is $p_{c}(n)= \begin{cases}1+\frac{2}{n-1}, & n \geq 2 \\ +\infty, & n=1\end{cases}$

Mathematics Subject Classification (2010). 35L05; 35L70.
\end{abstract}

Keywords. Damped wave equation, Critical exponent, Blow up, Global existence.

\section{Introduction}

We consider the Cauchy problem for the semilinear wave equation

$$
\begin{cases}u_{t t}-\Delta u+V(x) u_{t}=|u|^{p}, & (x, t) \in \mathbb{R}^{n} \times(0, \infty), \\ u(x, 0)=\epsilon u_{0}(x), u_{t}(x, 0)=\epsilon u_{1}(x), & x \in \mathbb{R}^{n},\end{cases}
$$

where $\epsilon>0, p$ satisfies

$$
1<p<+\infty(n=1,2), \quad 1<p \leq \frac{n}{n-2}(n \geq 3),
$$

$\left(u_{0}, u_{1}\right) \in H^{1} \times L^{2}$ satisfy: there exists a constant $R>0$, such that $\operatorname{supp}\left\{u_{0}, u_{1}\right\} \subset B_{R}(0)=\left\{x \in \mathbb{R}^{n}|| x \mid<R\right\}$, and $V(x) \in C\left(\mathbb{R}^{n}\right)$ is a potential function which will be specified later.

We focus on the critical exponent $p_{c}(n)$ of the problem $(1.1)$, which is a number defined by the following property:

If $p>p_{c}(n)$, then all solutions of (1.1) with small initial values are global; while if $1<p \leq p_{c}(n)$, then all solutions of (1.1) with nonnegative initial values blow up in finite time regardless of the smallness of the initial values.

When the potential $V(x)$ is a constant $(V(x) \equiv 1)$, Todorova and Yordanov [8] in 2001 obtained that the critical exponent of (1.1) is $1+\frac{2}{n}$, which is 
the same as the Fujita exponent for the heat equation $v_{t}-\Delta v=v^{p}$ (see Fujita [1] and Weissler [10]). More precisely, they proved that:

If $1+\frac{2}{n}<p \leq \frac{n}{n-2}(n \geq 3), 1+\frac{2}{n}<p<\infty(n=1,2)$, and the initial values with compact supports are sufficiently small, then the problem (1.1) admits a unique global solution

$$
u \in C\left([0, \infty), H^{1}\right) \cap C^{1}\left([0, \infty), L^{2}\right) ;
$$

While if $1<p<1+\frac{2}{n}, \int_{\mathbb{R}^{n}} u_{0}(x) d x>0, \int_{\mathbb{R}^{n}} u_{1}(x) d x>0$, then all the solutions to (1.1) blow up in finite time.

Later Zhang [11] proved that the critical exponent $1+\frac{2}{n}$ belongs to the blow up region. In 2005, Ikehata and Tanizawa [2] extended the global existence result of [8] to initial values without compact supports.

When $V(x) \in C^{1}\left(\mathbb{R}^{n}\right)$ is a radially symmetric function,

$$
V(x) \sim V_{0}(1+|x|)^{-\alpha},|x| \rightarrow \infty, \quad V_{0}>0, \alpha \in(0,1),
$$

Ikehata et al. [3] in 2009 obtained that the critical exponent for (1.1) is $1+\frac{2}{n-\alpha}$. More precisely, they proved that:

If $1+\frac{2}{n-\alpha}<p<\frac{n+2}{n-2}(n \geq 3), 1+\frac{2}{n-\alpha}<p<\infty(n=1,2)$, and the initial values are sufficiently small, then the problem (1.1) admits a unique global solution

$$
\begin{array}{r}
u \in C\left([0, \infty), H^{1}\right) \cap C^{1}\left([0, \infty), L^{2}\right), \\
\int_{\mathbb{R}^{n}}\left(\left|u_{t}\right|^{2}+|\nabla u|^{2}\right) d x \leq C(1+t)^{-\left(\frac{n-\alpha}{2-\alpha}+1-\delta\right),}
\end{array}
$$

where $\delta>0$ is an arbitrarily small number; While if

$$
1<p \leq 1+\frac{2}{n-\alpha}, \quad \int_{\mathbb{R}^{n}}\left(u_{1}+V(x) u_{0}\right) d x>0
$$

then all the solutions to (1.1) do not exist globally for any $\epsilon>0$.

In this paper we solve the critical exponent problem for (1.1) with critical potential $V(x)$. More precisely, we assume that $V(x) \in C\left(\mathbb{R}^{n}\right)$ satisfies:

$$
V_{0}\left(1+|x|^{2}\right)^{-1 / 2} \leq V(x) \leq V_{1}\left(1+|x|^{2}\right)^{-1 / 2}, \quad V_{0}, V_{1}>0 .
$$

To get the critical exponent we need the decay estimates for the homogeneous problem

$$
\begin{cases}u_{t t}-\Delta u+V(x) u_{t}=0, & (x, t) \in \mathbb{R}^{n} \times(0, \infty), \\ u(x, 0)=u_{0}(x), u_{t}(x, 0)=u_{1}(x), & x \in \mathbb{R}^{n},\end{cases}
$$

where $V(x)$ satisfies (1.2), and $\left(u_{0}, u_{1}\right) \in H^{1} \times L^{2}, \operatorname{supp}\left\{u_{0}, u_{1}\right\} \subset B_{R}(0)$. Matsumura [5], Mochizuki and Nakazawa [6], Uesaka [9] discussed the energy decay rate of the problem (1.3), and obtained

$$
\int_{\mathbb{R}^{n}}\left(u_{t}^{2}+|\nabla u|^{2}\right) d x \leq C(1+t)^{-\min \left\{V_{0}, 1\right\}} .
$$

Recently, Ikehata et al. [4] obtained that the solution to (1.3) satisfies: when $n=1,2$,

$$
\int_{\mathbb{R}^{n}}\left(u_{t}^{2}+|\nabla u|^{2}\right) d x \leq \begin{cases}C_{\delta} I_{0}^{2}(1+t)^{-V_{0}+\delta}, & 0<V_{0} \leq n \\ C I_{0}^{2}(1+t)^{-n}, & V_{0}>n\end{cases}
$$


when $n \geq 3$,

$$
\int_{\mathbb{R}^{n}}\left(u_{t}^{2}+|\nabla u|^{2}\right) d x \leq \begin{cases}C_{\delta} I_{0}^{2}(1+t)^{-V_{0}+\delta}, & 0<V_{0} \leq 1, \\ C I_{0}^{2}(1+t)^{-V_{0}}, & 1<V_{0}<n, \\ C_{\delta} I_{0}^{2}(1+t)^{-n+\delta}, & V_{0} \geq n,\end{cases}
$$

where $I_{0}=\left\|u_{0}\right\|_{H^{1}}+\left\|u_{1}\right\|_{2}, \delta>0$ is an arbitrarily small number. Using multiplier method, in another paper, we can prove that when $n=2$,

$$
\int_{\mathbb{R}^{n}}\left(u_{t}^{2}+|\nabla u|^{2}\right) d x \leq C I_{0}^{2}(1+t)^{-V_{0}}, \quad 1<V_{0} \leq 2 .
$$

Above all, the optimal estimates obtained for the solutions to (1.3) are as follows:

when $n=1,2$,

$$
\int_{\mathbb{R}^{n}}\left(u_{t}^{2}+|\nabla u|^{2}\right) d x \leq C I_{0}^{2}(1+t)^{-\min \left\{V_{0}, n\right\}},
$$

when $n \geq 3$,

$$
\int_{\mathbb{R}^{n}}\left(u_{t}^{2}+|\nabla u|^{2}\right) d x \leq \begin{cases}C I_{0}^{2}(1+t)^{-V_{0}}, & 0<V_{0}<n \\ C_{\delta} I_{0}^{2}(1+t)^{-n+\delta}, & V_{0} \geq n\end{cases}
$$

where $\delta>0$ is an arbitrarily small number.

Now we are ready to state our main results. The main result of this paper is that the critical exponent $p_{c}(n)$ for $(1.1)\left(V_{0} \geq n\right)$ is $p_{c}(n)=1+\frac{2}{n-1}$.

Our global existence result is as follows.

Theorem 1.1. Let $n \geq 2, V(x)$ satisfy (1.2), $V_{0} \geq n, 1+\frac{2}{n-1}<p<+\infty(n=$ $2)$, and $1+\frac{2}{n-1}<p \leq \frac{n}{n-2}(n \geq 3)$. Then there exists $\epsilon_{0}>0$ such that problem (1.1) admits a unique global solution

$$
u \in C\left([0, \infty), H^{1}\right) \cap C^{1}\left([0, \infty), L^{2}\right),
$$

for each $\epsilon<\epsilon_{0}$. Moreover, for any $\delta \in\left(0, \min \left\{\frac{1}{4}, \frac{(n-1) p-(n+1)}{2(p-1)}\right\}\right]$, the global solution satisfies

$$
\begin{array}{rr}
\|D u\|_{2} \leq C \epsilon\left(\left\|u_{0}\right\|_{H^{1}}+\left\|u_{1}\right\|_{2}\right)(1+t)^{-1}, & n=2, \\
\|D u\|_{2} \leq C \epsilon\left(\left\|u_{0}\right\|_{H^{1}}+\left\|u_{1}\right\|_{2}\right)(1+t)^{-\frac{n}{2}+\delta}, & n \geq 3,
\end{array}
$$

where $D=\left(\partial_{t}, \nabla_{x}\right), C>0$ is a constant, independent of $t$.

The blow up result is as follows.

Theorem 1.2. Let $V(x)$ satisfy (1.2), $1<p<\infty(n=1)$, and $1<p \leq$ $1+\frac{2}{n-1}(n \geq 2)$. If $V(x) u_{1}(x)+u_{0}(x) \geq 0$ and $V(x) u_{1}(x)+u_{0}(x) \not \equiv 0$, then the solution to (1.1) does not exist globally, for any $\epsilon>0$.

Remark 1.3. We did not find good methods to solve the critical exponent problem to (1.1) for $0<V_{0}<n$.

This paper is organized as follows. In Sect. 2 we prove Theorem 1.1 by dividing the proof into several lemmas. In Sect. 3 we prove Theorem 1.2. 


\section{Global existence result for small initial values}

The following local result for the problem (1.1) is well-known, which can be obtained by a simply modification of the result in Strauss [7].

Lemma 2.1. Let $V(x) \in C\left(\mathbb{R}^{n}\right)$ satisfy (1.2). Then the problem (1.1) admits a unique local solution $u \in C\left([0, T), H^{1}\right) \cap C^{1}\left([0, T), L^{2}\right)$ satisfying

$$
u(x, t) \equiv 0, \quad|x| \geq t+R,
$$

where $T>0$ depending only on $\|D u(0)\|_{2}$. Moreover, the solution can be continued beyond the interval $[0, T)$ if $\sup \|D u(t)\|_{2}<+\infty$.

In view of this local existence result, global existence of a solution follows the boundedness of the energy at all times. In the following, we prove Theorem 1.1 by the method of Todorova and Yordanov [8].

Choosing a weight function $\psi(x, t)=\frac{1+|x|}{1+t}$, we obtain the following weighted energy estimate.

Lemma 2.2. Let $V(x)$ satisfy (1.2), $V_{0} \geq 1, u(x, t)$ be a local solution to (1.1) on $[0, T)$, and $\psi(x, t)=\frac{1+|x|}{1+t}$. Then for any $t \in[0, T)$, the following estimate holds:

$$
\left\|e^{\psi} D u\right\|_{2} \leq C \epsilon+C\left(\max _{[0, t]}(s+1)^{\eta_{1}}\left\|e^{\gamma_{1} \psi(s)} u(s)\right\|_{p+1}\right)^{(p+1) / 2},
$$

where $\eta_{1}>0, \gamma_{1}>\frac{2}{p+1}$ are arbitrarily numbers, and $C>0$ is a constant, independent of $\epsilon$ and $t$.

Proof. Multiplying Eq. (1.1) by $e^{2 \psi} u_{t}$ and rearranging the terms, we obtain

$$
\begin{aligned}
& \left(e^{2 \psi} \frac{u_{t}^{2}+|\nabla u|^{2}}{2}\right)_{t}-\nabla\left(e^{2 \psi} u_{t} \nabla u\right)+\frac{e^{2 \psi}}{-\psi_{t}}\left|\psi_{t} \nabla u-u_{t} \nabla \psi\right|^{2} \\
& +\frac{e^{2 \psi}}{-\psi_{t}}\left(\psi_{t}^{2}-|\nabla \psi|^{2}-V(x) \psi_{t}\right) u_{t}^{2}=\left(\frac{e^{2 \psi}|u|^{p} u}{p+1}\right)_{t}-\frac{2 e^{2 \psi}|u|^{p} u \psi_{t}}{p+1}
\end{aligned}
$$

By the choice of $\psi(x, t)$ and $V_{0} \geq 1$, we obtain

$$
\begin{array}{r}
-\psi_{t}=\frac{1+|x|}{(1+t)^{2}}>0, \quad \psi_{x_{i}}=\frac{1}{1+t} \frac{x_{i}}{|x|}, \quad|\nabla \psi|^{2}=\frac{1}{(1+t)^{2}} \\
\psi_{t}^{2}-|\nabla \psi|^{2}-V(x) \psi_{t} \geq \frac{(1+|x|)^{2}}{(1+t)^{4}}-\frac{1}{(1+t)^{2}}+\frac{1+|x|}{(1+t)^{2}} \frac{V_{0}}{\sqrt{1+|x|^{2}}} \geq 0
\end{array}
$$

Thus, by the above arguments, (2.1) can be simplified to

$$
\left(e^{2 \psi} \frac{u_{t}^{2}+|\nabla u|^{2}}{2}\right)_{t}-\nabla\left(e^{2 \psi} u_{t} \nabla u\right) \leq\left(\frac{e^{2 \psi}|u|^{p} u}{p+1}\right)_{t}-\frac{2 e^{2 \psi}|u|^{p} u \psi_{t}}{p+1} .
$$


Integrating $(2.2)$ over $[0, t] \times \mathbb{R}^{n}$, and letting $\epsilon \leq 1$, we obtain

$$
\begin{aligned}
\left\|e^{\psi} D u\right\|_{2}^{2} \leq & C\left(\epsilon^{2}+\epsilon^{p+1}\right)+C \int_{\mathbb{R}^{n}} e^{2 \psi}|u|^{p+1} d x+C \int_{0}^{t} \int_{\mathbb{R}^{n}} e^{2 \psi}|u|^{p+1}\left|\psi_{s}\right| d x d s \\
\leq & C \epsilon^{2}+C\left\|e^{\frac{2 \psi}{p+1}} u\right\|_{p+1}^{p+1} \\
& +C \int_{0}^{t} \max _{\operatorname{supp} u(s)}\left(\left|\psi_{s}\right| e^{\left(2-\gamma_{1}(p+1)\right) \psi(s)}\right)\left\|e^{\gamma_{1} \psi(s)} u(s)\right\|_{p+1}^{p+1} d s,
\end{aligned}
$$

where $\gamma_{1}>\frac{2}{p+1}$, and $C>0$ is a constant, independent of $\epsilon$ and $t$. By the choice of $\psi(x, t)$, we have

$$
\begin{aligned}
\max _{\operatorname{supp} u(s)}\left|\psi_{s}\right| e^{\left(2-\gamma_{1}(p+1)\right) \psi(s)} & =\max _{|x| \leq s+R} \frac{1+|x|}{(1+s)^{2}} e^{\left(2-\gamma_{1}(p+1)\right) \psi(s)} \\
& \leq \frac{1+R+s}{(1+s)^{2}} \leq \frac{C}{1+s}
\end{aligned}
$$

Thus, we have

$$
\begin{aligned}
\left\|e^{\psi} D u\right\|_{2}^{2} & \leq C \epsilon^{2}+C\left\|e^{\frac{2 \psi}{p+1}} u\right\|_{p+1}^{p+1}+C \int_{0}^{t} \frac{1}{1+s}\left\|e^{\gamma_{1} \psi(s)} u(s)\right\|_{p+1}^{p+1} d s \\
& \leq C \epsilon^{2}+C\left\|e^{\frac{2 \psi}{p+1}} u\right\|_{p+1}^{p+1}+C\left[\max _{[0, t]}(1+s)^{\eta_{1}}\left\|e^{\gamma_{1} \psi(s)} u(s)\right\|_{p+1}\right]^{p+1} \\
& \leq C \epsilon^{2}+C\left[\max _{[0, t]}(1+s)^{\eta_{1}}\left\|e^{\gamma_{1} \psi(s)} u(s)\right\|_{p+1}\right]^{p+1}
\end{aligned}
$$

where $\eta_{1}>0$ is an arbitrarily number. We complete the proof of Lemma 2.2 .

Lemma 2.3. Let $n \geq 2, V(x)$ satisfy (1.2), $V_{0} \geq n, u(x, t)$ be a local solution to $(1.1)$ on $[0, T)$, and $\delta \in\left(0, \frac{1}{4}\right]$ is any fixed number. Then for any $t \in[0, T)$, the following estimates hold:

when $n=2$,

$$
\|D u(t)\|_{2} \leq C(1+t)^{-1}\left(\epsilon+\max _{[0, t]}\left[(1+s)^{\frac{1+\eta}{p}}\left\|e^{\gamma \psi(s)} u(s)\right\|_{2 p}\right]^{p}\right),
$$

when $n \geq 3$,

$$
\|D u(t)\|_{2} \leq C_{\delta}(1+t)^{-n / 2+\delta}\left(\epsilon+\max _{[0, t]}\left[(1+s)^{\frac{n / 2-\delta}{p}}\left\|e^{\gamma \psi(s)} u(s)\right\|_{2 p}\right]^{p}\right),
$$

where $\eta>0$ and $\gamma>0$ are arbitrarily small numbers.

Proof. Let $u_{L}(x, t)$ be the solution to the homogeneous problem

$$
\begin{cases}u_{t t}-\Delta u+V(x) u_{t}=0, & (x, t) \in \mathbb{R}^{n} \times(0, \infty), \\ u(x, 0)=\epsilon u_{0}(x), u_{t}(x, 0)=\epsilon u_{1}(x), & x \in \mathbb{R}^{n},\end{cases}
$$

and $S(t) * u_{1}(x)$ be the solution to the problem

$$
\begin{cases}u_{t t}-\Delta u+V(x) u_{t}=0, & (x, t) \in \mathbb{R}^{n} \times(0, \infty), \\ u(x, 0)=0, u_{t}(x, 0)=u_{1}(x), & x \in \mathbb{R}^{n}\end{cases}
$$


Then

$$
u(x, t)=u_{L}(x, t)+\int_{0}^{t} S(t-\tau) *|u|^{p}(\tau) d \tau
$$

is a solution to the problem (1.1). In the following, we split the proof of Lemma 2.3 into two cases.

Case $1(n=2)$. By the energy estimate (1.4) for problem (2.3), the linear term $\left\|D u_{L}(t)\right\|_{2}$ is bounded by

$$
\left\|D u_{L}(t)\right\|_{2} \leq C \epsilon(1+t)^{-1}\left(\left\|u_{0}\right\|_{H^{1}}+\left\|u_{1}\right\|_{2}\right) \leq C \epsilon(1+t)^{-1},
$$

and the integral term is estimated as follows:

$$
\begin{aligned}
& \left\|D\left(\int_{0}^{t} S(t-\tau) *|u|^{p}(\tau) d \tau\right)\right\|_{2} \leq \int_{0}^{t}\left\|D S(t-\tau) *|u|^{p}(\tau)\right\|_{2} d \tau \\
& \quad \leq C \int_{0}^{t}(1+t-\tau)^{-1}\left\|u^{p}(\tau)\right\|_{2} d \tau=C \int_{0}^{t}(1+t-\tau)^{-1}\|u(\tau)\|_{2 p}^{p} d \tau .
\end{aligned}
$$

Since $\psi(x, t)>0$, for any $\gamma>0$, we have

$$
\|u(\tau)\|_{2 p} \leq\left\|e^{\gamma \psi(\tau)} u(\tau)\right\|_{2 p} .
$$

Inserting (2.6) into (2.5) and splitting the integral into two parts, we have

$$
\begin{aligned}
& \left\|D\left(\int_{0}^{t} S(t-\tau) *|u|^{p}(\tau) d \tau\right)\right\|_{2} \\
& \quad \leq C\left(\int_{0}^{t / 2}+\int_{t / 2}^{t}\right)(1+t-\tau)^{-1}\left\|e^{\gamma \psi(\tau)} u(\tau)\right\|_{2 p}^{p} d \tau=C\left(I_{1}+I_{2}\right) .
\end{aligned}
$$

For any $\eta>0$,

$$
\begin{gathered}
I_{1}=\int_{0}^{t / 2}(1+t-\tau)^{-1} \frac{1}{(1+\tau)^{1+\eta}}(1+\tau)^{1+\eta}\left\|e^{\gamma \psi(\tau)} u(\tau)\right\|_{2 p}^{p} d \tau \\
\leq C(1+t)^{-1} \max _{[0, t / 2]}\left[(1+\tau)^{(1+\eta) / p}\left\|e^{\gamma \psi(\tau)} u(\tau)\right\|_{2 p}\right]^{p} \\
I_{2}=\int_{t / 2}^{t}(1+\tau)^{-1}(1+t-\tau)^{-(1+\eta)}(1+t-\tau)^{\eta}(1+\tau)\left\|e^{\gamma \psi(\tau)} u(\tau)\right\|_{2 p}^{p} d \tau \\
\leq C(1+t)^{-1} \max _{[t / 2, t]}\left[(1+\tau)^{(1+\eta) / p}\left\|e^{\gamma \psi(\tau)} u(\tau)\right\|_{2 p}\right]^{p}
\end{gathered}
$$

Combining (2.4), (2.7), (2.8) and (2.9), we obtain, when $n=2$,

$$
\|D u(t)\|_{2} \leq C(1+t)^{-1}\left(\epsilon+\max _{[0, t]}\left[(1+s)^{\frac{1+\eta}{p}}\left\|e^{\gamma \psi(s)} u(s)\right\|_{2 p}\right]^{p}\right) .
$$

Case $2(n \geq 3)$. By the energy estimate (1.5) for problem (2.3), the linear term $\left\|D u_{L}(t)\right\|_{2}$ is bounded by

$$
\left\|D u_{L}(t)\right\|_{2} \leq C_{\delta} \epsilon\left(\left\|u_{0}\right\|_{H^{1}}+\left\|u_{1}\right\|_{2}\right)(1+t)^{-n / 2+\delta},
$$


and the integral term is estimated as follows:

$$
\begin{aligned}
& \left\|D\left(\int_{0}^{t} S(t-\tau) *|u|^{p}(\tau) d \tau\right)\right\|_{2} \leq \int_{0}^{t}\left\|D S(t-\tau) *|u|^{p}(\tau)\right\|_{2} d \tau \\
& \leq C_{\delta} \int_{0}^{t}(1+t-\tau)^{-n / 2+\delta}\left\|u^{p}(\tau)\right\|_{2} d \tau=C_{\delta} \int_{0}^{t}(1+t-\tau)^{-n / 2+\delta}\|u(\tau)\|_{2 p}^{p} d \tau \\
& \leq C_{\delta}\left(\int_{0}^{t / 2}+\int_{t / 2}^{t}\right)(1+t-\tau)^{-n / 2+\delta}\left\|e^{\gamma \psi(\tau)} u(\tau)\right\|_{2 p}^{p} d \tau=C_{\delta}\left(I_{3}+I_{4}\right)
\end{aligned}
$$

For any $\eta>0$,

$$
\begin{aligned}
I_{3} & =\int_{0}^{t / 2}(1+t-\tau)^{-n / 2+\delta} \frac{1}{(1+\tau)^{1+\eta}}(1+\tau)^{1+\eta}\left\|e^{\gamma \psi(\tau)} u(\tau)\right\|_{2 p}^{p} d \tau \\
& \leq C(1+t)^{-n / 2+\delta} \max _{[0, t / 2]}\left[(1+\tau)^{(1+\eta) / p}\left\|e^{\gamma \psi(\tau)} u(\tau)\right\|_{2 p}\right]^{p} \\
I_{4} & =\int_{t / 2}^{t}(1+\tau)^{-n / 2+\delta}(1+t-\tau)^{-n / 2+\delta}(1+\tau)^{n / 2-\delta}\left\|e^{\gamma \psi(\tau)} u(\tau)\right\|_{2 p}^{p} d \tau \\
\leq & C(1+t)^{-n / 2+\delta} \max _{[t / 2, t]}\left[(1+\tau)^{(n / 2-\delta) / p}\left\|e^{\gamma \psi(\tau)} u(\tau)\right\|_{2 p}\right]^{p} .
\end{aligned}
$$

Combining (2.10)-(2.13), we obtain, when $n \geq 3$,

$$
\|D u(t)\|_{2} \leq C_{\delta}(1+t)^{-n / 2+\delta}\left(\epsilon+\max _{[0, t]}\left[(1+s)^{\frac{n / 2-\delta}{p}}\left\|e^{\gamma \psi(s)} u(s)\right\|_{2 p}\right]^{p}\right),
$$

which completes the proof of Lemma 2.3.

Lemma 2.4. Let $\sigma \in(0,1], 2 \leq q<\infty(n=2), 2 \leq q \leq \frac{2 n}{n-2}(n \geq 3), \psi(x, t)=$ $\frac{1+|x|}{1+t}, u(x, t)$ be a local solution of (1.1) on $[0, T)$. Then for any $t \in[0, T)$, the following estimate holds:

$$
\left\|e^{\sigma \psi(t)} u\right\|_{q} \leq C(1+t)^{1-\theta(q)}\left\|e^{\psi(t)} \nabla u\right\|_{2}^{\sigma}\|\nabla u\|_{2}^{1-\sigma},
$$

where $\theta(q)=n\left(\frac{1}{2}-\frac{1}{q}\right)$, and $C>0$ is a constant, independent of $t$.

Proof. Applying the Gagliardo-Nirenberg inequality to $e^{\sigma \psi} u$, we have

$$
\left\|e^{\sigma \psi} u\right\|_{q} \leq\left\|e^{\sigma \psi} u\right\|_{2}^{1-\theta(q)}\left\|\nabla\left(e^{\sigma \psi} u\right)\right\|_{2}^{\theta(q)} .
$$

On the other hand,

$$
e^{\sigma \psi} \nabla u=\nabla\left(e^{\sigma \psi} u\right)-\sigma e^{\sigma \psi} u \nabla \psi
$$

Thus,

$$
\begin{aligned}
\left\|e^{\sigma \psi} \nabla u\right\|_{2}^{2} & =\int_{\mathbb{R}^{n}}\left(\left|\nabla\left(e^{\sigma \psi} u\right)\right|^{2}+\sigma^{2} e^{2 \sigma \psi}|u|^{2}|\nabla \psi|^{2}-2 \sigma e^{\sigma \psi} u \nabla\left(e^{\sigma \psi} u\right) \nabla \psi\right) d x \\
& =\int_{\mathbb{R}^{n}}\left(\left|\nabla\left(e^{\sigma \psi} u\right)\right|^{2}+\sigma^{2} e^{2 \sigma \psi}|u|^{2}|\nabla \psi|^{2}+\sigma \Delta \psi\left(e^{\sigma \psi} u\right)^{2}\right) d x
\end{aligned}
$$

By the choice of $\psi(x, t)$, we have

$$
|\nabla \psi|^{2}=\frac{1}{(1+t)^{2}}, \quad \Delta \psi=\frac{n-1}{1+t} \frac{1}{|x|} .
$$


Inserting (2.16) into (2.15), we have

$$
\left\|e^{\sigma \psi} \nabla u\right\|_{2}^{2} \geq\left\|\nabla\left(e^{\sigma \psi} u\right)\right\|_{2}^{2}+\frac{\sigma^{2}}{(1+t)^{2}}\left\|e^{\sigma \psi} u\right\|_{2}^{2} .
$$

By (2.17), (2.14) can be simplified to

$$
\left\|e^{\sigma \psi} u\right\|_{q} \leq C(\sigma)(1+t)^{1-\theta(q)}\left\|e^{\sigma \psi} \nabla u\right\|_{2} .
$$

Using Hölder inequality,

$$
\begin{aligned}
\left\|e^{\sigma \psi} \nabla u\right\|_{2}^{2} & =\int_{\mathbb{R}^{n}} e^{2 \sigma \psi}|\nabla u|^{2 \sigma}|\nabla u|^{2(1-\sigma)} d x \\
& \leq\left(\int_{\mathbb{R}^{n}} e^{2 \psi}|\nabla u|^{2}\right)^{\sigma}\left(\int_{\mathbb{R}^{n}}|\nabla u|^{2}\right)^{1-\sigma} .
\end{aligned}
$$

Thus,

$$
\left\|e^{\sigma \psi} \nabla u\right\|_{2} \leq\left\|e^{\psi} \nabla u\right\|_{2}^{\sigma}\|\nabla u\|_{2}^{1-\sigma} .
$$

Inserting (2.19) into (2.18), we complete the proof of Lemma 2.4.

Now we are ready to prove Theorem 1.1.

Proof of Theorem 1.1. We split the proof into two cases.

Case $1(n=2)$. We introduce the weighted energy functional

$$
W(t)=\left\|e^{\psi} D u\right\|_{2}+(1+t)\|D u\|_{2} .
$$

By Lemmas 2.2 and 2.3, we have

$$
\begin{aligned}
W(t) \leq & C \epsilon+C\left(\max _{[0, t]}(s+1)^{\eta_{1}}\left\|e^{\gamma_{1} \psi(s)} u(s)\right\|_{p+1}\right)^{(p+1) / 2} \\
& +C\left[\max _{[0, t]}(1+s)^{\frac{1+\eta}{p}}\left\|e^{\gamma \psi(s)} u(s)\right\|_{2 p}\right]^{p}
\end{aligned}
$$

where $\eta>0, \gamma>0, \eta_{1}>0, \gamma_{1}>\frac{2}{p+1}$ are arbitrarily small numbers. By Lemma 2.4 and the definition of $W(t)$, we obtain

$$
\begin{aligned}
\left\|e^{\gamma_{1} \psi(s)} u(s)\right\|_{p+1} & \leq C(1+s)^{1-\theta(p+1)}\left\|e^{\psi(s)} \nabla u(s)\right\|_{2}^{\gamma_{1}}\|\nabla u(s)\|_{2}^{1-\gamma_{1}} \\
& \leq C(1+s)^{1-\theta(p+1)-\left(1-\gamma_{1}\right)} W(s), \\
\left\|e^{\gamma \psi(s)} u(s)\right\|_{2 p} & \leq C(1+s)^{1-\theta(2 p)}\left\|e^{\psi(s)} \nabla u(s)\right\|_{2}^{\gamma}\|\nabla u(s)\|_{2}^{1-\gamma} \\
& \leq C(1+s)^{1-\theta(2 p)-(1-\gamma)} W(s),
\end{aligned}
$$

where $\theta(p+1)=n\left(\frac{1}{2}-\frac{1}{p+1}\right), \theta(2 p)=n\left(\frac{1}{2}-\frac{1}{2 p}\right)$. Using (2.21) and (2.22), we obtain from $(2.20)$

$$
\begin{aligned}
W(t) \leq & C \epsilon+C \max _{[0, t]}(s+1)^{\frac{p+1}{2} \eta_{1}+\frac{p+1}{2}\left[1-\theta(p+1)-\left(1-\gamma_{1}\right)\right]} W(s)^{(p+1) / 2} \\
& +C \max _{[0, t]}(1+s)^{1+\eta+p[1-\theta(2 p)-(1-\gamma)]} W(s)^{p}
\end{aligned}
$$


In the following we calculate the exponents of $(1+s)$ in $(2.23)$. Set $\gamma_{1}=$ $\frac{2}{p+1}+\eta_{2}, \eta_{2}>0$, then

$$
\begin{gathered}
\frac{p+1}{2} \eta_{1}+\frac{p+1}{2}\left[1-\theta(p+1)-\left(1-\gamma_{1}\right)\right]=\frac{p+1}{2}\left(\eta_{1}+\eta_{2}\right)+\frac{3-p}{2} \\
1+\eta+p[1-\theta(2 p)-(1-\gamma)]=\eta+p \gamma+(2-p)
\end{gathered}
$$

Since $p>3$, we can choose $\eta>0, \lambda>0, \eta_{1}>0, \eta_{2}>0$ sufficiently small, such that the exponents of $(1+s)$ are negative. Thus we have from (2.23)

$$
W(t) \leq C \epsilon+C \max _{[0, t]} W(s)^{\frac{p+1}{2}}+C \max _{[0, t]} W(s)^{p} .
$$

Set $M(t)=\max _{[0, t]} W(s)$. From $(2.24)$, we have

$$
M(t) \leq C \epsilon,
$$

for sufficiently small $\epsilon$, which completes the proof of Theorem 1.1 for $n=2$.

Case $2(n \geq 3)$. For any fixed $\delta \in\left(0, \min \left\{\frac{1}{4}, \frac{(n-1) p-(n+1)}{2(p-1)}\right\}\right]$, we introduce the weighted energy functional

$$
W_{1}(t)=\left\|e^{\psi} D u\right\|_{2}+(1+t)^{\frac{n}{2}-\delta}\|D u\|_{2} .
$$

By Lemmas 2.2 and 2.3, we have

$$
\begin{aligned}
W_{1}(t) \leq & C \epsilon+C\left(\max _{[0, t]}(s+1)^{\eta_{1}}\left\|e^{\gamma_{1} \psi(s)} u(s)\right\|_{p+1}\right)^{(p+1) / 2} \\
& +C_{\delta}\left[\max _{[0, t]}(1+s)^{\frac{n / 2-\delta}{p}}\left\|e^{\gamma \psi(s)} u(s)\right\|_{2 p}\right]^{p}
\end{aligned}
$$

where $\gamma>0, \eta_{1}>0, \gamma_{1}>\frac{2}{p+1}$ are arbitrarily small numbers. By Lemma 2.4 and the definition of $W_{1}(t)$, we obtain

$$
\begin{aligned}
\left\|e^{\gamma_{1} \psi(s)} u(s)\right\|_{p+1} & \leq C(1+s)^{1-\theta(p+1)}\left\|e^{\psi(s)} \nabla u(s)\right\|_{2}^{\gamma_{1}}\|\nabla u(s)\|_{2}^{1-\gamma_{1}} \\
& \leq C(1+s)^{1-\theta(p+1)-(n / 2-\delta)\left(1-\gamma_{1}\right)} W_{1}(s) \\
\left\|e^{\gamma \psi(s)} u(s)\right\|_{2 p} & \leq C(1+s)^{1-\theta(2 p)}\left\|e^{\psi(s)} \nabla u(s)\right\|_{2}^{\gamma}\|\nabla u(s)\|_{2}^{1-\gamma} \\
& \leq C(1+s)^{1-\theta(2 p)-(n / 2-\delta)(1-\gamma)} W_{1}(s)
\end{aligned}
$$

where $\theta(p+1)=n\left(\frac{1}{2}-\frac{1}{p+1}\right), \theta(2 p)=n\left(\frac{1}{2}-\frac{1}{2 p}\right)$. Using (2.26) and (2.27), we obtain from $(2.25)$

$$
\begin{aligned}
W_{1}(t) \leq & C \epsilon+C \max _{[0, t]}(s+1)^{\frac{p+1}{2} \eta_{1}+\frac{p+1}{2}\left[1-\theta(p+1)-(n / 2-\delta)\left(1-\gamma_{1}\right)\right]} W_{1}(s)^{(p+1) / 2} \\
& +C \max _{[0, t]}(1+s)^{n / 2-\delta+p[1-\theta(2 p)-(n / 2-\delta)(1-\gamma)]} W_{1}(s)^{p}
\end{aligned}
$$


In the following we calculate the exponents of $(1+s)$ in $(2.28)$. Set $\gamma_{1}=$ $\frac{2}{p+1}+\eta_{2}, \eta_{2}>0$, then

$$
\begin{aligned}
& \frac{p+1}{2} \eta_{1}+\frac{p+1}{2}\left[1-\theta(p+1)-(n / 2-\delta)\left(1-\gamma_{1}\right)\right] \\
& \quad=\frac{p+1}{2}\left(\eta_{1}+\eta_{2}(n / 2-\delta)\right)+\frac{p-1}{2} \delta+\frac{1}{2}[n+1-(n-1) p], \\
& n / 2-\delta+p[1-\theta(2 p)-(n / 2-\delta)(1-\gamma)] \\
& \quad=(n / 2-\delta) \gamma p+(p-1) \delta+[n-(n-1) p] .
\end{aligned}
$$

Since $p>1+\frac{2}{n-1}, \delta \in\left(0, \min \left\{\frac{1}{4}, \frac{(n-1) p-(n+1)}{2(p-1)}\right\}\right]$, we have

$$
\frac{p-1}{2} \delta+\frac{1}{2}[n+1-(n-1) p]<0, \quad(p-1) \delta+[n-(n-1) p]<0 .
$$

Choose $\gamma>0, \eta_{1}>0, \eta_{2}>0$ sufficiently small, such that the exponents of $(1+s)$ are negative. Thus we have from $(2.28)$

$$
W_{1}(t) \leq C \epsilon+C \max _{[0, t]} W_{1}(s)^{\frac{p+1}{2}}+C \max _{[0, t]} W_{1}(s)^{p} .
$$

Set $M_{1}(t)=\max _{[0, t]} W_{1}(s)$. From $(2.29)$, we have

$$
M_{1}(t) \leq C \epsilon,
$$

for sufficiently small $\epsilon$, which completes the proof of Theorem 1.1 for $n \geq 3$.

\section{Blow up result}

In this section we prove Theorem 1.2 by the method of test functions (see Zhang [11]).

Proof of Theorem 1.2. Choose a function $\phi(s) \in C^{\infty}([0, \infty))$ satisfying

$$
\begin{gathered}
\phi(s)=\left\{\begin{array}{ll}
1, & s<1, \\
0, & s>2,
\end{array} \quad 0 \leq \phi(s) \leq 1,\right. \\
\left|\phi^{\prime}(s)\right| \leq C \phi^{\frac{1}{p}}(s), \quad\left|\phi^{\prime \prime}(s)\right| \leq C \phi^{\frac{1}{p}}(s),
\end{gathered}
$$

where $C>0$ is a constant. Set $\varphi(x, t)=\phi(|x|) \phi(t)$. For any $L>0$, define

$$
\varphi_{L}(x, t)=\varphi\left(\frac{x}{L}, \frac{t}{L}\right), \quad(x, t) \in \mathbb{R}^{n} \times[0, \infty) .
$$

In the following, we prove Theorem 1.2 by contradiction. Suppose $u(x, t) \in$ $C\left([0, \infty), H^{1}\right) \cap C^{1}\left([0, \infty), L^{2}\right)$ is a global solution to (1.1). Multiplying equation $(1.1)$ by $\varphi_{L}(x, t)$ and integrating the corresponding equality by parts over $\mathbb{R}^{n} \times[0, \infty)$, we obtain

$$
\begin{aligned}
\int_{0}^{\infty} & \int_{\mathbb{R}^{n}} u\left(\partial_{t t} \varphi_{L}-\Delta \varphi_{L}-V(x) \partial_{t} \varphi_{L}\right) d x d t \\
& -\int_{\mathbb{R}^{n}}\left(u_{1}(x) \varphi_{L}(x, 0)-u_{0}(x) \partial_{t} \varphi_{L}(x, 0)+V(x) u_{0}(x) \varphi_{L}(x, 0)\right) d x \\
= & \int_{0}^{\infty} \int_{\mathbb{R}^{n}} \varphi_{L}|u|^{p} d x d t .
\end{aligned}
$$


By the choice of $\varphi_{L}(x, t)$, we have

$$
\varphi_{L}(x, 0)=\phi\left(\frac{|x|}{L}\right) \geq 0, \quad \partial_{t} \varphi_{L}(x, 0) \equiv 0 .
$$

By the assumption $u_{1}(x)+V(x) u_{0}(x) \geq 0$, we obtain from (3.1)

$$
\int_{0}^{\infty} \int_{\mathbb{R}^{n}} \varphi_{L}|u|^{p} d x d t \leq \int_{0}^{\infty} \int_{\mathbb{R}^{n}} u\left(\partial_{t t} \varphi_{L}-\Delta \varphi_{L}-V(x) \partial_{t} \varphi_{L}\right) d x d t
$$

Using Hölder inequality, we estimate the integral on the right in (3.2)

$$
\begin{aligned}
\int_{0}^{\infty} & \int_{\mathbb{R}^{n}} u\left(\partial_{t t} \varphi_{L}-\Delta \varphi_{L}-V(x) \partial_{t} \varphi_{L}\right) d x d t \\
\leq & C\left(\int_{L}^{2 L} \int_{B_{2 L}(0)}|u|^{p} \varphi_{L} d x d t+\int_{0}^{2 L} \int_{B_{2 L}(0) \backslash B_{L}(0)}|u|^{p} \varphi_{L} d x d t\right)^{1 / p} \\
& \times\left(\int_{0}^{\infty} \int_{\mathbb{R}^{n}} \varphi_{L}^{-p^{\prime} / p}\left|\partial_{t t} \varphi_{L}-\Delta \varphi_{L}-V(x) \partial_{t} \varphi_{L}\right|^{p^{\prime}} d x d t\right)^{1 / p^{\prime}}
\end{aligned}
$$

where $p^{\prime}=p /(p-1)$. By the properties of $\varphi_{L}(x, t)$, we obtain

$$
\begin{aligned}
\int_{0}^{\infty} & \int_{\mathbb{R}^{n}} \varphi_{L}^{-p^{\prime} / p}\left|\partial_{t t} \varphi_{L}-\Delta \varphi_{L}-V(x) \partial_{t} \varphi_{L}\right|^{p^{\prime}} d x d t \\
= & \int_{0}^{\infty} \int_{\mathbb{R}^{n}} \varphi^{-p^{\prime} / p}\left(\frac{x}{L}, \frac{t}{L}\right) \mid \frac{1}{L^{2}} \varphi_{t t}\left(\frac{x}{L}, \frac{t}{L}\right)-\frac{1}{L^{2}} \Delta \varphi\left(\frac{x}{L}, \frac{t}{L}\right) \\
& -\left.V(x) \frac{1}{L} \varphi_{t}\left(\frac{x}{L}, \frac{t}{L}\right)\right|^{p^{\prime}} d x d t \\
\leq & \frac{C}{L^{2 p^{\prime}}} \int_{0}^{2 L} \int_{B_{2 L}(0)} d x d t+\frac{C}{L^{p^{\prime}}} \int_{0}^{2 L} \int_{B_{2 L}(0)}|V(x)|^{p^{\prime}} d x d t \\
\leq & C L^{n+1-2 p^{\prime}}+C L^{1-p^{\prime}} \int_{0}^{2 L} \frac{(1+r)^{n-1}}{(1+r)^{p^{\prime}}} d r \\
\leq & \begin{cases}C L^{n+1-2 p^{\prime}}, & n-p^{\prime}>0, \\
C L^{1-p^{\prime}} \ln L, & n-p^{\prime}=0, \\
C L^{1-p^{\prime}}, & n-p^{\prime}<0 .\end{cases}
\end{aligned}
$$

Inserting (3.3) and (3.4) into (3.2), we obtain

$$
\begin{aligned}
& \left(\int_{0}^{\infty} \int_{\mathbb{R}^{n}} \varphi_{L}|u|^{p} d x d t\right)^{p /(p-1)} \\
& \leq C\left(\int_{L}^{2 L} \int_{B_{2 L}(0)}|u|^{p} \varphi_{L} d x d t+\int_{0}^{2 L} \int_{B_{2 L}(0) \backslash B_{L}(0)}|u|^{p} \varphi_{L} d x d t\right)^{1 /(p-1)} \\
& \quad \times \begin{cases}C L^{n+1-2 p^{\prime}}, & n-p^{\prime}>0 \\
C L^{1-p^{\prime}} \ln L, & n-p^{\prime}=0 \\
C L^{1-p^{\prime}}, & n-p^{\prime}<0 .\end{cases}
\end{aligned}
$$

Finally, we show that the above inequality can not hold as $L \rightarrow \infty$. 
Case 1. When $1<p<1+\frac{2}{n-1}(n \geq 2), 1<p<\infty(n=1)$, the exponents of $L$ in (3.5) are negative. In (3.5), letting $L \rightarrow \infty$, we obtain

$$
\int_{0}^{\infty} \int_{\mathbb{R}^{n}}|u|^{p} d x d t \leq 0,
$$

which is impossible, since $u$ is a nontrivial solution.

Case 2. When $p=1+\frac{2}{n-1}(n \geq 2)$, the exponents of $L$ in (3.5) are nonpositive. In (3.5), letting $T \rightarrow \infty$, we obtain

$$
\int_{0}^{\infty} \int_{\mathbb{R}^{n}}|u|^{p} d x d t \leq C .
$$

In (3.5), letting $T \rightarrow \infty$, and considering (3.6), we obtain

$$
\int_{0}^{\infty} \int_{\mathbb{R}^{n}}|u|^{p} d x d t \leq 0
$$

which is impossible, since $u$ is a nontrivial solution. By Case 1 and Case 2, we complete the proof of Theorem 1.2.

\section{References}

[1] Fujita, H.: On the blowing up of solutions to the Cauchy problem for $u_{t}=$ $\Delta u+u^{1+\alpha}$, . J. Fac. Sci. Univ. Tokyo Sect. I 13, 109-124 (1966)

[2] Ikehata, R., Tanizawa, K.: Global existence of solutions for semilinear damped wave equations in $\mathbf{R}^{N}$ with noncompactly supported initial data. Nonlinear Anal. 61, 1189-1208 (2005)

[3] Ikehata, R., Todorova, G., Yordanov, B.: Critical exponent for semilinear wave equations with space-dependent potential. Funkcialaj Ekvacioj 52, 411435 (2009)

[4] Ikehata, R., Todorova, G., Yordanov, B.: Optimal decay rate of the energy for wave equations with critical potential (2012, preprint)

[5] Matsumura, A.: Energy decay of solutions of dissipative wave equations. Proc. Japan Acad. Ser. A 53, 232-236 (1977)

[6] Mochizuki, K., Nakazawa, H.: Energy decay and asymptotic behavior of solutions to the wave equations with linear dissipation. Publ. RIMS. Kyoto Univ. 32, 401-414 (1996)

[7] Strauss, W.A.: Nonlinear wave equations, in: CBMS Regional Conference Series in Math., vol. 73, AMS, Providence, ISBN: 0-8218-0725-0, 1989, x+91 pp. Published for the conference board of the Math. Sci. Washington, D.C.

[8] Todorova, G., Yordanov, B.: Critical exponent for a nonlinear wave equation with damping. J. Differ. Equ. 174, 464-489 (2001)

[9] Uesaka, B.: The total energy decay of solutions for the wave equation with a dissipative term. J. Math. Kyoto Univ. 20(1), 57-65 (1979) 
[10] Weissler, F.: Existence and non-existence of global solutions for a semilinear heat equation. Israel J. Math. 38, 29-40 (1981)

[11] Zhang, Q.S.: A blow-up result for a nonlinear wave equation with damping: the critical case. C. R. Acad. Sci. Paris Sér. I 333, 109-114 (2001)

\section{Xinfu Li}

School of Science

Tianjin University of Commerce

Tianjin 300134

China

e-mail: lxf123465@pku.edu.cn

Received: 4 May 2012.

Accepted: 6 December 2012. 